\title{
Der Musterentwurf für ein Landesstrafvollzugsgesetz als Konsequenz des Phlegmas um die Europäischen Strafvoll- zugsgrundsätze?
}

Nina Nestler

\section{Einführung}

Längst hat die Europäisierung des Strafrechts neben den Bereichen von Strafverfolgung und materiellem Strafrecht den Strafvollzug erreicht. Hier normieren Mindeststandards unter anderem die Europäischen Strafvollzugsgrundsätze in Gestalt von (nicht verpflichtenden) Empfehlungen des Europarats für seine Mitgliedstaaten, welche die Verhältnisse im staatlich angeordneten Freiheitsentzug betreffen. Im Hinblick auf die Vorgaben etwa der Europäischen Menschenrechtskonvention oder der CPT-Standards sollen die European Prison Rules deren Inhalte umfassend konkretisieren. Viel diskutiert und weitgehend als positiv eingeschätzt ${ }^{1}$, bleibt indes wegen ihrer fehlenden Verbindlichkeit fraglich und durchaus umstritten, inwieweit den Europäischen Gefängnisregeln tatsächlich eine dem Umfang des Rekurses gerecht werdende Relevanz zukommt.

Die Europäischen Gefängnisregeln von 1973 basieren auf den Mindestgrundsätzen der Vereinten Nationen für die Behandlung von Gefangenen aus dem Jahr 1955 (so genannte Minima der Vereinten Nationen). Eine überarbeitete Fassung wurde zunächst 1987 beschlossen, bevor nach weiteren Neuerungen das Ministerkomitee des Europarats die Europäischen Strafvollzugsgrundsätze 2006 zum 11. Januar 2006 als „Recommendation Rec (2006) 2 on the European Prison Rules“ in ihrer heutigen Fassung verabschiedete ${ }^{2}$. $\mathrm{Zu}$ Beginn legen die Europäischen Gefängnisregeln in Teil I „Basic Principles“ fest, fokussieren jedoch nicht nur die Menschenwürde, sondern die Beachtung der Menschenrechte insgesamt. Die weiteren Teile beziehen sich detailliert auf Haftbedingungen mit Sonderregelungen für bestimmte Gefangenengruppen, die Bereiche der Gesundheitsfürsorge, Sicherheit und Ordnung, das Anstaltspersonal, Inspektion und Aufsicht, Untersuchungshaft sowie rechtskräftig verurteilte Strafgefangene. Allerdings begründen die Europäischen Strafvollzugsgrundsätze keine subjektiven Rechte und Pflichten des Gefangenen ${ }^{3}$. Die European Prison Rules, denen es an Verbindlichkeit fehlt, gelten damit als „weiches Recht“ (soft law), was ihre zumindest moralische Verbindlichkeit andeutet, während sie jedoch weder die nationalen Gerichte noch den Europäischen Gerichtshof für Menschenrechte binden ${ }^{4}$.

Am 6. September 2011 haben nun zehn Bundesländer ${ }^{5}$ einen $\mathrm{Mu}$ sterentwurf für ein Landesstrafvollzugsgesetz vorgelegt. Für diesen versichert die mit der Erstellung des Entwurfs befasste Arbeitsgruppe schon am Anfang ihrer Entwurfsbegründung, die Empfehlung Rec (2006) 2 des Ministerkomitees an die Mitgliedstaaten über die Europäischen Strafvollzugsgrundsätze sei beachtet worden ${ }^{6}$. Es gilt nachfolgend zu analysieren, inwieweit dies tatsächlich geschehen ist.

\section{Einhaltung der European Prison Rules durch den Musterentwurf}
1. Ausdrückliche Bezugnahme auf die European Prison Rules

Auf die Europäischen Strafvollzugsgrundsätze verweist die Entwurfsbegründung nur in einigen wenigen Fällen explizit.

\section{a) Grundprinzipien}

Die neun Grundprinzipien der Europäischen Strafvollzugsgrundsätzen schreiben unter anderem vor, alle Inhaftierten unter Achtung ihrer Menschenrechte zu behandeln und, dass in Unfreiheit befindliche Personen ihrer Rechte nicht verlustig gehen, welche ihnen nicht wirksam durch die Verhängung der Freiheitsstrafe oder der Untersuchungshaft entzogen wurden. Damit legen die Grundprinzipien die essentialia fest - das Grundgerüst, an dem die Haftbedingungen insgesamt ausgerichtet sein sollen ${ }^{7}$. Auf diese Prinzipien nimmt die Entwurfsbegründung an keiner Stelle Bezug. Selbst im Zusammenhang mit der Ausrichtung des Vollzugs auf eine Wiedereingliederung des Inhaftierten (vgl. Nr. 6 EPR) findet ein Rekurs auf jene Basic Principles nicht statt.

\section{b) Haftbedingungen}

Die Europäischen Gefängnisregeln enthalten sodann in Nrn. 10 bis 108 EPR detaillierte Bestimmungen zu den Haftbedingungen. Zu denselben Materien schlägt auch der Musterentwurf Regelungen vor; seine Begründung nimmt aber nur an wenigen Stellen Bezug auf die European Prison Rules. Auf Nr. 102.1 EPR ${ }^{8}$ rekurriert der Entwurf bspw. in der Begründung zu $\ 2$ Satz $1 \mathrm{ME}-L S t$ VollzG, der als alleiniges Vollzugsziel die Resozialisierung festschreibt ${ }^{9}$. Der Entwurf führt hier neben den Europäischen Gefängnisregeln aber zusätzlich Nr. 65 der VN-Mindestgrundsätze für die Behandlung der Gefangenen sowie insbesondere das deutsche Verfassungsrecht an. Denn vor allem aus dem Grundgesetz selbst ergibt sich die Pflicht zur Achtung der Menschenwürde nach Art. 1 Abs. 1 GG und des Grundsatzes der Verhältnismäßigkeit. Im Bezug auf das Vollzugsziel der Resozialisierung entspricht der Entwurf außerdem - ohne dies in der Begründung zu erwähnen - Nr. 6 EPR $^{10}$, wonach die Freiheitsentziehung so durchzuführen ist, dass sie die Wiedereingliederung des Inhaftierten erleichtert.

\10 Satz 1 ME-LStVollzG normiert den Trennungsgrundsatz für männliche und weibliche Gefangene. Die Begründung führt hier Nr. 18.8b EPR ${ }^{11}$ an, wonach insbesondere zum Schutz weiblicher Gefangener vor Übergriffen eine Trennung notwendig ist ${ }^{12}$.

\19 ME-LStVollzG sieht arbeitstherapeutische Maßnahmen vor, die dazu dienen, den Gefangenen Eigenschaften wie Selbstvertrauen, Durchhaltevermögen und Konzentrationsfähigkeit einzuüben. Hierzu verweist die Begründung des Musterentwurfs auf Nr. 26.1 EPR ${ }^{13}$, 
wonach Gefangenenarbeit als ein positiver Bestandteil des Strafvollzugs betrachtet werden soll ${ }^{14}$. Sowohl das aktuelle Strafvollzugsgesetz als auch der Musterentwurf differenzieren zwischen Arbeit und arbeitstherapeutischen Maßnahmen, die Begründung des Entwurfs rekurriert indes nur im Bezug auf letztere auf die European Prison Rules.

$\mathrm{Zu} \ 27$ Nr. 3 ME-LStVollzG, welcher die Untersagung von Besuchen regelt, führt die Arbeitsgruppe der Länder Nr. 24.2 EPR ${ }^{15}$ an, der den Schutz von durch die Straftat betroffenen Personen bei der Durchführung von Besuchen im Blick hat ${ }^{16}$.

Bei der Begründung zu $\int 86$ ME-LStVollzG, welcher die Verhängung von Disziplinarmaßnahmen betrifft, wird auf Nr. 60.5 EPR $^{17}$ verwiesen. Dies beschränkt sich jedoch auf $\$ 86$ Abs. 3 ME-LStVollzG, der vorschreibt, dass Arrest nur wegen schwerer oder wiederholter Verfehlungen verhängt werden darf und mithin auf „absolute Ausnahmefälle zu beschränken “ ist ${ }^{18}$.

Für $\ 89$ ME-LStVollzG, welcher das Disziplinarverfahren regelt, beruft sich die Entwurfsbegründung hinsichtlich von Abs. 2 der Norm auf Nr. 56.2 EPR ${ }^{19}$. 889 Abs. 2 ME-LStVollzG schreibt vor, dass eine einvernehmliche Streitbeilegung stattfinden kann. Dadurch soll der Begründung entsprechend einem zeitgemäßen Verständnis von Konfliktlösung Rechnung getragen werden, wonach „mit den Gefangenen in geeigneten Fällen Gespräche mit dem Ziel des Abschlusses einer Vereinbarung geführt werden können "20. Mit den dadurch intendierten positiven Effekten einer aktiven Mitwirkung an der Konfliktlösung trägt die Regelung Nr. 56.2 EPR Rechnung.

Weitere ausdrückliche Bezugnahmen existieren in der Begründung des Musterentwurfs nicht.

\section{Inhaltlich mit den European Prison Rules korrespondie- rende Regelungen}

Im Übrigen existieren zwar etliche Regelungen, die mit den European Prison Rules übereinstimmen, für welche die Entwurfsbegründung auf jene Grundsätze aber nicht ausdrücklich Bezug nimmt.

\section{a) Grundprinzipien}

So verhält es sich mit Nr. 2 EPR $^{21}$, die vorsieht, dass Menschen im Freiheitsentzug alle (übrigen) Rechte behalten, die ihnen nicht durch die Entscheidung, mit der gegen sie eine Freiheitsstrafe verhängt oder Untersuchungshaft angeordnet wurde, entzogen sind. Die Normierung dieses Grundsatzes offenbart sich bei korrekter Anwendung des deutschen (Strafvollzugs-) Rechts aber letztlich als nicht erforderlich. Denn es folgt schon aus dem Wesen eines Rechts, dass es seinem Inhaber so lange zusteht, bis es entweder durch Hoheitsakt rechtmäßig entzogen oder verwirkt wird, bzw. sein Inhaber - soweit jenes disponibel ist - darauf verzichtet. $\int 4 \mathrm{ME}-\mathrm{LStV}$ VollzG trägt dem vergleichbar mit $\$ 4$ StVollzG Rechnung, welcher die Rechtsstellung des Inhaftierten sichert. Zudem regelt $\mathbb{} 106$ ME-LStVollzG, inhaltlich identisch mit $\mathbb{} 196$ StVollzG, welche Grundrechte durch den Strafvollzug eingeschränkt werden.

Nr. 5 EPR ${ }^{22}$ enthält den Angleichungsgrundsatz, wonach das Leben in der Vollzugsanstalt demjenigen außerhalb anzunähern ist. Diesbezüglich korrespondieren die European Prison Rules mit dem $\mathrm{Mu}-$ sterentwurf, der z.B. in $\$ 3$ Abs. 3 ME-LStVollzG gebietet, das Leben im Vollzug den allgemeinen Lebensverhältnissen so weit wie möglich anzugleichen. Zusätzlich normiert $\ 3$ Abs. 4 ME-LStVollzG sogar den Gegensteuerungsgrundsatz, demgemäß schädlichen Folgen des
Freiheitsentzuges entgegenzuwirken ist. Ferner stimmt das Gebot mit der Judikatur des Bundesverfassungsgerichts und der obergerichtlichen Rechtsprechung zum Strafvollzugsgesetz überein, die bereits mehrfach, in verschiedenen Zusammenhängen auf den Angleichungsgrundsatz verwiesen haben ${ }^{23}$.

Mit den Grundsätzen der Nrn. $6^{24}$ und $7^{25}$ EPR korrespondiert die bundesgesetzliche Regelung des $\$ 154$ StVollzG, welcher die Zusammenarbeit mit externen sozialen Diensten als obligatorisch normiert und insofern auf die Wiedereingliederung des Inhaftierten in die Gesellschaft abzielt. Der Musterentwurf kennt eine $\mathbb{1} 154$ StVollzG entsprechende Regelung nicht, schreibt jedoch - gemäß der Vorgabe der Nr. 7 EPR - in $\$ 21$ Abs. 4 ME-LStVollzG vor, bei der Vollzugsplanung darauf zu achten, dass die Gefangenen Qualifizierungsmaßnahmen während ihrer Haftzeit beenden oder im Anschluss daran fortsetzen können. Hier besteht zwar eine inhaltliche Übereinstimmung, die Entwurfsbegründung erwähnt dies jedoch nicht explizit.

Nr. 8 EPR $^{26}$ sieht die Einhaltung hoher Standards bei Auswahl und Ausbildung des Anstaltspersonals sowie deren Arbeitsbedingungen vor. Eine ähnliche Regelung kennt auch der Musterentwurf mit $\$ 96$ ME-LStVollzG, der vorschreibt, dass die Anstalt mit dem für die Erreichung des Vollzugsziels und die Erfüllung ihrer Aufgaben erforderlichen Personal ausgestattet wird. Die Entwurfsbegründung zu dieser inhaltlich weniger detailreichen Norm verweist dazu jedoch nicht auf Nr. 8 EPR, was zu einer diesbezüglichen Konkretisierung hätte beitragen können.

Auch im Bezug auf Nr. 9 EPR ${ }^{27}$, der eine Kontrolle durch staatliche Stellen vorsieht, scheint der Musterentwurf der Empfehlung zu entsprechen. Denn zum einen führen gemäß $\mathbb{1 0 1}$ Abs. 1 ME-LStVollzG ( $\$ 151$ Abs. 1 Satz 1 StVollzG) die Landesjustizverwaltungen Aufsicht über die Justizvollzugsanstalten. Zum anderen besteht für den Inhaftierten nach $\mathbb{\int} 109$ ff. StVollzG, $\mathbb{\int} 119$ a StPO sowie $\mathbb{S} 92$ JGG die Möglichkeit, einzelne Maßnahmen einer justiziellen Überprüfung zu unterwerfen.

\section{b) Haftbedingungen}

Das strikte Gebot der Einzelunterbringung Gefangener während der Ruhezeit gilt selbst nach Nr. $18.5 \mathrm{EPR}^{28}$ nur, sofern nicht die gemeinschaftliche Unterbringung sinnvoller erscheint. In Nr. 18.6 EPR ${ }^{29}$ wird dies dahingehend konkretisiert, dass die Zellen stets eine hinreichende Größe für die Belegung mit mehreren Häftlingen aufweisen müssen und zudem ausschließlich mit solchen Inhaftierten belegt werden dürfen, die sich für die gemeinsame Unterbringung eignen. Auch $\ 11$ Abs. 1 ME-LStVollzG sowie $\ 18$ Abs. 1 Satz 1 StVollzG sehen während der Ruhezeit vor, Gefangene allein in ihren Hafträumen unterzubringen. \18 Abs. 1 Satz 2 StVollzG lässt aber, ohne dabei ein Zustimmungserfordernis zu erwähnen, die gemeinsame Unterbringung nur zu, sofern ein Gefangener hilfsbedürftig ist oder eine Gefahr für dessen Leben oder Gesundheit besteht - mithin die gemeinschaftliche Unterbringung deswegen sinnvoller erscheint. Demgegenüber erlaubt $₫ 11$ Abs. 2 Satz 1 ME-LStVollzG die gemeinsame Unterbringung grds. nur bei Zustimmung der Inhaftierten. Beiden Vorschriften ist jedoch gemein, dass sie bei Hilfsbedürftigkeit die gemeinsame Unterbringung erlauben; der Musterentwurf trifft hierzu lediglich eine ausdrückliche und gesonderte Bestimmung in \11 Abs. 2 Satz 2 ME-LStVollzG. Die Regelung kann, selbst wenn dies in der Begründung keine explizite Erwähnung findet, durchaus als Konkretisierung des in Nr. 18.6 EPR enthaltenen Gedankens verstanden werden. 
Nr. 60.4 EPR ${ }^{30}$ normiert die Ermöglichung von Familienkontakten selbst während der Vollstreckung von Disziplinarmaßnahmen. Zwar enthält der Musterentwurf keine derartige explizite Regelung; lediglich $\$ 26$ Abs. 2 ME-LStVollzG sieht vor, Besuche von Angehörigen im Sinne von $\mathbb{1} 11$ Abs. 1 Nr. 1 StGB besonders zu unterstützen. Auch nimmt $\mathbb{2} 27$ Nr. 2 ME-LStVollzG Angehörige von einem Besuchsverbot wegen der Befürchtung eines schädlichen Einflusses aus und $\mathbb{3} 2 \mathrm{Nr}$. 2 ME-LStVollzG gestattet unter gleichen Voraussetzungen den Schriftwechsel mit jenen Personen. Mit Nr. 60.4 EPR konform geht jedoch $\ 86$ Abs. 2 ME-LStVollzG, der den Katalog zulässiger Disziplinarmaßnahmen enthält, und - anders als dies in \ 103 Abs. 1 Nr. 8 StVollzG vorgesehen ist - keine Beschränkung des Verkehrs mit Personen außerhalb der Anstalt kennt. In der Entwurfsbegründung heißt es dazu allerdings nur, die Beschränkung von Außenkontakten sei ebenfalls nicht mehr vorgesehen, da „sie von besonderer Bedeutung für die Aufrechterhaltung sozialer Bindungen sind" ${ }^{31}$. Hinsichtlich von Nr. 60.4 EPR fehlt indes jede Bezugnahme, obwohl sich eigentlich ein Hinweis an dieser Stelle geradezu aufdrängt.

Ähnlich verhält es sich mit Nr. 93.1 EPR ${ }^{32}$, welche die Überwachung der Haftbedingungen und der Behandlung von Gefangenen durch unabhängige Gremien in veröffentlichten Untersuchungsberichten vorsieht. $\int 92$ Abs. 2 ME-LStVollzG regelt in diesem Zusammenhang Evaluation sowie kriminologische Forschung und schreibt zu diesem Zweck in seinem Abs. 1 vor, Behandlungsprogramme. Nach Abs. 2 der Norm soll der Strafvollzug, dabei insbesondere seine Aufgabenerfüllung und Gestaltung, die Umsetzung seiner Leitlinien sowie die Behandlungsprogramme und deren Wirkungen auf die Erreichung des Vollzugsziels, regelmäßig durch den kriminologischen Dienst, eine Hochschule oder eine andere Stelle wissenschaftlich begleitet und erforscht werden. Damit entspricht der Musterentwurf Nr. 93.1 EPR; die Entwurfsbegründung beruft sich dabei allerdings nicht auf die Europäischen Gefängnisregeln, sondern vielmehr auf das Resozialisierungskonzept, welches dem Entwurf zu Grunde $\operatorname{liegt}{ }^{33}$.

\section{Inhaltliche Übereinstimmung mit den European Prison Rules trotz fehlender Regelung}

Schließlich enthalten die European Prison Rules Vorgaben, zu denen der Musterentwurf zwar überhaupt keine Regelung trifft; eine solche ist jedoch nicht erforderlich, weil im deutschen Recht bereits andere Rechtssätze die zwingende Einhaltung dieser Anforderungen vorgeben.

\section{a) Grundprinzipien}

So verhält es sich mit der Achtung der Menschenwürde, die Nr. 1 EPR $^{34}$ vorgibt. Dies korrespondiert mit Art. 1 Abs. 1 GG und bedarf eigentlich keiner weiteren expliziten Erwähnung. Im Gegenteil scheint der Umstand, dass es für erforderlich gehalten wird, dies in jedem denkbaren Kontext gesondert festzuschreiben, sogar geeignet, die Selbstverständlichkeit dieses Grundsatzes zu beeinträchtigen. Denn das Grundgesetz beim Wort nehmend duldet der Terminus der Unantastbarkeit in schlechthin keinem Zusammenhang eine Einschränkung - eben auch nicht im Fall staatlich angeordneter Freiheitsentziehung. Insofern hätte es zumindest aus deutscher Sicht der Nr. 1 EPR nicht bedurft ${ }^{35}$. Daher bringt auch die Entwurfsbegründung die Menschenwürde an denjenigen Stellen, an denen sie erwähnt wird, nicht in Verbindung mit den European Prison Rules. ${ }^{36}$
Gemäß Nr. 3 EPR ${ }^{37}$ sind Beschränkungen des Inhaftierten immer auf das Mindestmaß zu reduzieren und müssen im Bezug auf den Zweck der Freiheitsentziehung verhältnismäßig sein. Dieser Grundsatz und insbesondere das darin zum Ausdruck kommende Übermaßverbot sind dem deutschen Recht aber keineswegs fremd. Vielmehr stellt das Verhältnismäßigkeitsprinzip ein wesentliches Merkmal des Rechtsstaats dar ${ }^{38}$. Als verfassungsrechtliches Gebot ist der Grundsatz der Verhältnismäßigkeit gemäß Art. 1 Abs. 3, Art. 20 Abs. 3 GG für die gesamte Staatsgewalt - einschließlich der den Freiheitsentzug anordnenden und vollziehenden Stellen - unmittelbar verbindlich. Damit überein stimmt die einschlägige Rechtsprechung des Bundesverfassungsgerichts, der zufolge Einschränkungen auf das Mindestmaß begrenzt sein, nur einen rechtmäßigen Zweck verfolgen und dem Verhältnismäßigkeitsgrundsatz auch i. e. S. entsprechen müs$\operatorname{sen}^{39}$.

Nach Nr. 4 EPR ${ }^{40}$ stellt Mittelknappheit keine Rechtfertigung für Vollzugsbedingungen dar, die gegen Menschenrechte verstoßen. Diese Übersetzung des Originaltextes wirkt zunächst schief, da ein Verstoß niemals rechtmäßig bzw. „gerechtfertigt“ erfolgt; gemeint sein kann hier vielmehr nur eine Beschränkung von Menschenrechten aufgrund begrenzter finanzieller Ressourcen ${ }^{41}$. Auch hierfür bietet jedoch bereits das in der Bundesrepublik Deutschland geltende Recht einen Anknüpfungspunkt. So muss sich jede Beschränkung von (Menschen-)Rechten zunächst am Verhältnismäßigkeitsprinzip messen lassen, wo eine Prüfung regelmäßig ergeben wird, dass es für den Eingriff schon an der Erforderlichkeit fehlt. Jedenfalls dürfte spätestens auf der Ebene der Angemessenheit bzw. der Verhältnismäßigkeit i. e. S. das angestrebte Ziel finanzieller Einsparungen unproportional zu dem gewählten Mittel der Einschränkung von Menschenrechten sein. Die absolute Grenze zulässiger Beschränkungen bildet schließlich Art. 1 Abs. 1 GG. Mit dem in Nr. 4 EPR normierten Grundsatz stimmt zudem die Rechtsprechung des Bundesverfassungsgerichts überein, das bereits in verschiedenem Kontext - etwa in Entscheidungen zur Größe von Hafträumen und zur Mehrfachbelegung ${ }^{42}$ - wiederholt betonte, dass sich menschenrechtswidrige Vollzugsbedingungen durch Mittelknappheit nicht legitimieren lassen.

\section{b) Haftbedingungen}

Auch die Standards der Nrn. 14 bis 108 EPR sind im deutschen Recht weitgehend konkretisiert - entweder durch das Gesetz oder durch obergerichtliche Rechtsprechung - weshalb der Musterentwurf hierzu auch grds. keine ausdifferenzierte Regelung enthalten muss. Ohnehin erscheint zweifelhaft, ob detailreichere Vorschriften zu begrüßen sind, oder ob man sich jedenfalls im Bereich ganz prinzipieller Fragen eher an offene und daher flexiblere Klauseln halten sollte.

Dies gilt beispielsweise in Bezug auf das Gebot heimatnaher Unterbringung der Nr. 17.1 EPR ${ }^{43}$. $\$ 16$ ME-LStVollzG normiert, entsprechend $\ 8$ StVollzG, hier zwar lediglich abstrakt die Möglichkeit von Verlegungen, welche der Erreichung des Vollzugsziels der Wiedereingliederung, mithin - wie es $\ 8$ Abs. $1 \mathrm{Nr} .1$ StVollzG formuliert der Behandlung des Gefangenen oder seiner Eingliederung nach der Entlassung, förderlich sein müssen. Jedoch präzisierte das Bundesverfassungsgericht zumindest $\ 8$ StVollzG dahingehend, dass bei der Überprüfung eines Antrags auf Anstaltswechsel die Verlegung eines Strafgefangenen zur Aufrechterhaltung familiärer Beziehungen nicht erst dann in Betracht kommt, wenn dies als Behandlungsmaß- 
nahme oder zur Resozialisierung unerlässlich erscheint. Für das Resozialisierungsziel, auf das der Strafvollzug von Verfassungs wegen auszurichten ist, haben familiäre Beziehungen des Gefangenen vielmehr eine ganz wesentliche Bedeutung. Die Förderung des Kontakts zu den Angehörigen bleibt deshalb als entscheidender Gesichtspunkt in die Ermessensausübung im Bezug auf die beantragte Verlegung in eine heimatnähere Anstalt einzubeziehen ${ }^{44}$. Eine heimatnahe Unterbringung stellt somit einen Behandlungsfaktor von essentieller Bedeutung dar und muss deswegen so weit wie möglich sichergestellt sein. Da der Musterentwurf gerade diesem Aspekt besonders gerecht werden wollte ${ }^{45}$, ist davon auszugehen, dass sich an dieser Einschätzung zu $\ 8$ StVollzG auch für $\ 16$ ME-LStVollzG trotz der fehlenden expliziten Regenlung auch künftig nichts ändern soll.

Auch für die Festlegung der Mindestgröße von Hafträumen (Nr. 18.3 EPR ${ }^{46}$ ) existieren Vorgaben durch die Rechtsprechung. So stellt etwa das OLG Hamm einen Verstoß gegen die Menschenwürde fest, wenn bei gemeinschaftlicher Unterbringung mehrerer Gefangener in einer Zelle - selbst bei deren Ausstattung mit einer räumlich abgetrennten, gesondert entlüfteten Toilette - auf den einzelnen Gefangenen umgerechnet eine Grundfläche von weniger als $5 \mathrm{~m}^{2}$ entfällt ${ }^{47}$. In diesem Zusammenhang führt das Gericht allerdings zugleich aus, die Europäischen Strafvollzugsgrundsätze können die Rechtswidrigkeit einer solchen Unterbringung nicht begründen, da sie als bloße Empfehlungen des Europarats keine innerstaatliche Gesetzeskraft haben und den Anwendungsbereich des $\mathbb{S} 102$ Abs. 3 Satz 1 StVollzG nicht einschränken ${ }^{48}$. Nichts anderes wird insofern für $\mathbb{S} 86$ Abs. 5 ME-LStVollzG gelten, der mit $\mathbb{} 102$ Abs. 3 Satz 1 StVollzG korrespondiert.

\section{Zurückbleiben des Musterentwurfs hinter den Anforderungen der European Prison Rules?}

Der Musterentwurf für ein Strafvollzugsgesetz bleibt in Ermangelung einer ausdrücklichen Regelung sowie anderweitiger hinreichender Konkretisierung wohl nur an wenigen Stellen tatsächlich hinter den Empfehlungen der European Prison Rules zurück. So kennen die Strafvollzugsgrundsätze in Nr. 20.1 EPR $^{49}$ ein Recht der Häftlinge, mit angemessener Kleidung ausgestattet zu werden, sofern sie hierüber nicht selbst verfügen. Strafvollzugsgesetz und Musterentwurf normieren zunächst in $\$ 20$ StVollzG, $\mathbb{S} 52$ Abs. 1 ME-LStVollzG übereinstimmend, dass der Gefangene Anstaltskleidung trägt. Mit Nr. 20.4 EPR ${ }^{50}$ geht es konform, wenn gemäß $\mathbb{2 0}$ Abs. 2 Satz 1 StVollzG der Anstaltsleiter dem Gefangenen bei einer Ausführung gestatten muss, eigene Kleidung zu tragen, sofern kein Fluchtversuch droht. Auch darüber hinaus kann nach $\ 20$ Abs. 2 Satz 2 StVollzG dem Inhaftierten das Tragen eigener Kleidung gestattet werden, sofern er für Reinigung, Instandsetzung und regelmäßigen Wechsel auf eigene Kosten sorgt. Die bundesdeutsche Regelung steht somit nicht im Widerspruch zu den Europäischen Strafvollzugsgrundsätzen.

Eine solche explizite Ausnahme fehlt allerdings in dem Musterentwurf. Dieser erlaubt zwar eine abweichende Regelung durch den Anstaltsleiter, $\mathbb{} 52$ Abs. 2 Satz 1 ME-LStVollzG; überdies spricht \52 Abs. 2 Satz 2 ME-LStVollzG davon, dass der Gefangene für die Reinigung eigener Kleidung selbst zu sorgen hat, was den Schluss zulässt, dass deren Tragen prinzipiell gestattet werden kann. Ausdrücklich vorgesehen ist in dem Musterentwurf jedoch nicht, dass beim Verlassen der Anstalt neutrale Kleidung getragen werden darf, sondern die Entwurfsbegründung schweigt zu dieser Abweichung. Es bleibt aber fraglich, ob sich mit hinreichender Sicherheit sagen lässt, unter welchen genauen Voraussetzungen dem Inhaftierten ein
Anspruch aus Art. 1 Abs. 1 GG bzw. dem allgemeinen Persönlichkeitsrecht ${ }^{51}$ entsteht, außerhalb der Mauern nicht als Gefangener identifiziert zu werden.

\section{Fazit: Fehlende Signalwirkung}

Insgesamt wirkt die Bezugnahme auf die Europäischen Gefängnisregeln in der Begründung des Musterentwurfs eher sporadisch als konsequent. So erschließt sich nicht, weshalb in der Entwurfsbegründung an der einen Stelle ohne Argumentationsnot und ohne Konkretisierungsbedarf der Verweis auf die European Prison Rules geführt wird, während an anderer Stelle eine solche Bezugnahme fehlt, obwohl die Recommendation eine einschlägige Empfehlung beinhaltet. Der Musterentwurf der Länder scheint damit den Kurs fortzusetzen, sich mit fragmentarischen Verweisungen auf die European Prison Rules zu begnügen und es bei der bloß gelegentlichen Andeutung einer inhaltlichen Übereinstimmung zu belassen - dies bezeugt aber allenfalls guten Willen, jedoch keineswegs ein tatsächliches Aufbauen auf jene Vorgaben.

Dennoch bleibt der Musterentwurf für ein Strafvollzugsgesetz - von den aufgezeigten Zweifelsfällen abgesehen - hinter den Vorgaben der Europäischen Strafvollzugsgrundsätze nicht (wesentlich) zurück. Er ist jedoch weniger detailreich abgefasst und belässt es bei der Normierung allgemeinerer Grundsätze. Den fehlenden zwingenden Charakter kompensierte in Deutschland das BVerfG in einer Entscheidung aus dem Jahr 2006, indem es Verstöße gegen internationale Standards mit Menschenrechtsbezug als Indiz für eine unzureichende Gewichtung der Belange von Inhaftierten einordnete ${ }^{52}$. Das Gericht brachte dadurch mittelbar die internationalen aber unverbindlichen Normen zu einer gewissen Geltung - zumindest innerhalb der Bundesrepublik Deutschland. Wie aufgezeigt ist die Einhaltung der in den Europäischen Vollzugsgrundsätzen normierten Standards aber ohnehin auch weitgehend ohne Rekurs auf internationale Vorgaben gewährleistet. Insofern ist es besonders bedauerlich, dass der deutsche Musterentwurf für ein Landesstrafvollzugsgesetz nach außen hin und für andere Staaten erkennbar kein deutlicheres Signal ihrer Beachtung setzt.

\section{Fußnoten:}

1 Statt vieler Callies/Müller-Dietz, StVollzG, 11. Aufl. 2008, Einl. Rn. 59; Pollähne, StV 2007, S. 557 f.

$2 \mathrm{Im}$ Internet abrufbar unter https://wcd.coe.int/wcd/ViewDoc. jsp?id=955747.

3 Ostendorf, ZRP 2008, S. 14; Walter, ZJJ 2007, S. 74.

4 Vgl. Arloth, StVollzG, 3. Aufl. 2011, Einl. Rn. 11; Callies/Müller-Dietz, StVollzG, 11. Aufl. 2008, Einl. Rn. 59.

5 Berlin, Brandenburg, Bremen, Mecklenburg-Vorpommern, RheinlandPfalz, Saarland, Sachsen, Sachsen-Anhalt, Schleswig-Holstein und Thüringen.

6 ME-LStVollzG, Begründung, S. 65.

7 Callies/Müller-Dietz, StVollzG, 11. Aufl. 2008, Einl. Rn. 59.

8 In addition to the rules that apply to all prisoners, the regime for sentenced prisoners shall be designed to enable them to lead a responsible and crimefree life.

9 ME-LStVollzG, Begründung, S. 67.

10 All detention shall be managed so as to facilitate the reintegration into free society of persons who have been deprived of their liberty.

11 In deciding to accommodate prisoners in particular prisons or in particular sections of a prison due account shall be taken of the need to detain: male prisoners separately from females.

12 ME-LStVollzG, Begründung, S. 80.

13 Prison work shall be approached as a positive element of the prison regime and shall never be used as a punishment.

14 ME-LStVollzG, Begründung, S. 89.

15 Communication and visits may be subject to restrictions and monitoring necessary for the requirements of continuing criminal investigations, maintenance of good order, safety and security, prevention of criminal offences 
and protection of victims of crime, but such restrictions, including specific restrictions ordered by a judicial authority, shall nevertheless allow an acceptable minimum level of contact.

16 ME-LStVollzG, Begründung, S. 97.

17 Solitary confinement shall be imposed as a punishment only in exceptional cases and for a specified period of time, which shall be as short as possible.

18 ME-LStVollzG, Begründung, S. 151.

19 Whenever possible, prison authorities shall use mechanisms of restoration and mediation to resolve disputes with and among prisoners.

20 ME-LStVollzG, Begründung, S. 153.

21 Persons deprived of their liberty retain all rights that are not lawfully taken away by the decision sentencing them or remanding them in custody.

22 Life in prison shall approximate as closely as possible the positive aspects of life in the community.

23 So statt vieler bspw. BVerfG, Beschl. v. 15.07.2010, Az.: 2 BvR 328/07; KG Berlin, NStZ-RR 1996, S. 383 im Zusammenhang mit den Kosten für das Telefonieren.

24 All detention shall be managed so as to facilitate the reintegration into free society of persons who have been deprived of their liberty.

25 Co-operation with outside social services and as far as possible the involvement of civil society in prison life shall be encouraged.

26 Prison staff carry out an important public service and their recruitment, training and conditions of work shall enable them to maintain high standards in their care of prisoners.

27 All prisons shall be subject to regular government inspection and independent monitoring.

28 Prisoners shall normally be accommodated during the night in individual cells except where it is preferable for them to share sleeping accommodation.

29 Accommodation shall only be shared if it is suitable for this purpose and shall be occupied by prisoners suitable to associate with each other.

30 Punishment shall not include a total prohibition on family contact.

31 ME-LStVollzG, Begründung, S. 151.
32 The conditions of detention and the treatment of prisoners shall be monitored by an independent body or bodies whose findings shall be made public.

33 ME-LStVollzG, Begründung, S. 157.

34 All persons deprived of their liberty shall be treated with respect for their human rights.

35 So auch Laubenthal, in: FS-Scheuing, S. 364.

36 Bspw. ME-LStVollzG, Begründung, S. 67, 143.

37 Restrictions placed on persons deprived of their liberty shall be the minimum necessary and proportionate to the legitimate objective for which they are imposed.

38 Ausführlich Huster/Rux, in: BeckOK-GG, 11. Aufl. 2011, Art. 20 Rn. 176 ff.

39 BVerfGE 33, S. 1.

40 Prison conditions that infringe prisoners' human rights are not justified by lack of resources.

41 Laubenthal, in: FS-Scheuing, S. 365.

42 Vgl. hierzu BVerfG, NJW 2002, $2699 \mathrm{f}$.

43 Prisoners shall be allocated, as far as possible, to prisons close to their homes or places of social rehabilitation.

44 BVerfG, NStZ-RR 2006, S. 325 f.

45 ME-LStVollzG, Begründung, S. 67.

46 Specific minimum requirements in respect of the matters referred to in paragraphs 1 and 2 shall be set in national law.

47 OLG Hamm; NJW-RR 2008, S. 1408.

48 OLG Hamm, NJW-RR 2008, S. 1406.

49 Prisoners who do not have adequate clothing of their own shall be provided with clothing suitable for the climate.

50 Prisoners who obtain permission to go outside prison shall not be required to wear clothing that identifies them as prisoners.

51 BVerfG, NStZ 2000, S. 166.

52 BVerfG, ZJJ 2006, S. 193.

\title{
Grenzen der Vorfeldkriminalisierung
}

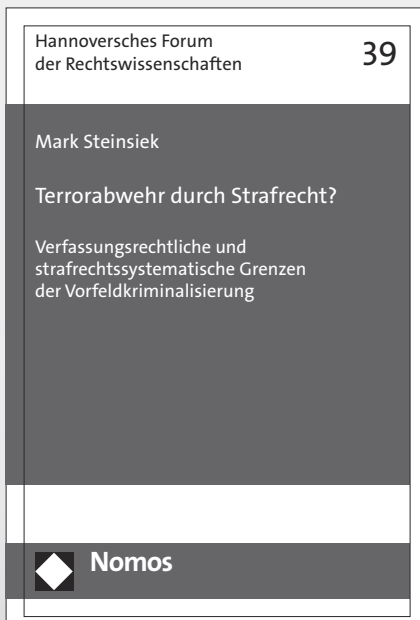

\author{
Terrorabwehr durch Strafrecht? \\ Verfassungsrechtliche und strafrechtssystematische \\ Grenzen der Vorfeldkriminalisierung \\ Von RR Dr. Mark Steinsiek
}

2012, 470 S., brosch., 99,- €, ISBN 978-3-8329-7522-7

(Hannoversches Forum der Rechtswissenschaften, Bd. 39)

Die Bedrohung der westlichen Welt durch den internationalen Terrorismus war das sicherheitsrechtlich bedeutsamste Thema des beginnenden Jahrtausends.

Das Werk arbeitet vor dem Hintergrund aktueller Terrorismusgesetzgebung ( $\$ \S 89 a, b, 91$ StGB) Tendenzen einer Vorverlagerung von Strafbarkeiten weit in das Vorfeld möglicher Rechtsgutsverletzungen heraus und zeigt die verfassungsrechtlichen und strafrechtsdogmatischen Grenzen umfassend auf. 